\title{
A THIRD GIANT PLANET ORBITING HIP 14810
}

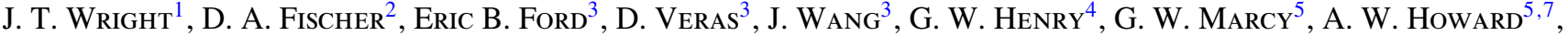 \\ AND JoHn Asher Johnson 6,8 \\ ${ }^{1}$ Department of Astronomy, 226 Space Sciences Building, Cornell University, Ithaca, NY 14853, USA; jtwright@ astro.cornell.edu \\ 2 Department of Physics and Astronomy, San Francisco State University, San Francisco, CA 94132, USA \\ ${ }^{3}$ Department of Astronomy, University of Florida, 211 Bryant Space Science Center, P.O. Box 112055, Gainesville, FL 32611-2055, USA \\ ${ }^{4}$ Center of Excellence in Information Systems, Tennessee State University, 3500 John A. Merritt Blvd., Box 9501, Nashville, TN 37209, USA \\ ${ }^{5}$ Department of Astronomy, 601 Campbell Hall, University of California, Berkeley, CA 94720-3411, USA \\ ${ }^{6}$ Institute for Astronomy, University of Hawai'i, Honolulu, HI 96822, USA \\ Received 2009 April 23; accepted 2009 June 2; published 2009 June 19
}

\section{ABSTRACT}

\begin{abstract}
We present new precision radial velocities and a three-planet Keplerian orbit fit for the $V=8.5$, G5 V star HIP 14810. We began observing this star at Keck Observatory as part of the N2K Planet Search Project. Wright et al. announced the inner two planets to this system, and subsequent observations have revealed the outer planet and the proper orbital solution for the middle planet. The planets have minimum masses of $3.9,1.3$, and $0.6 M_{\text {Jup }}$ and orbital periods of 6.67, 147.7, and 952 day, respectively. We have numerically integrated the family of orbital solutions consistent with the data and find that they are stable for at least $10^{6} \mathrm{yr}$. Our photometric search shows that the inner planet does not transit.
\end{abstract}

Key words: planetary systems - stars: individual (HIP 14810)

\section{INTRODUCTION}

The first multiple exoplanet system detected around a normal $\operatorname{star}^{9}$ was the triple system $v$ Andromadae (Butler et al. 1999). Today, over 30 systems comprising more than one planet are known (Wright et al. 2009), including seven triple systems, two quadruple systems ( $\mu$ Arae (Pepe et al. 2007) and GJ 581 (M. Mayor et al. 2009, submitted)), and the quintuple system 55 Cancri (Fischer et al. 2008).

Individual multiplanet systems offer insights into the dynamical evolution of planetary systems that singleton systems cannot. For instance, Ford et al. (2005) showed that $v$ Andromedae bears the scars of strong planet-planet scattering events preserved in its planets' orbital parameters. Other systems show evidence of migration and eccentricity pumping through mean-motion resonances (MMRs), which may be the signpost of convergent migration in multiplanet systems (e.g., Kley et al. 2004). Multiplanet systems with planet-planet interactions strong enough to be detected at current RV precision (as in GJ 876, Rivera et al. 2005) can allow for measurement of the inclination of the system, providing true planet masses.

Comparison of multiplanet systems as an ensemble to apparently singleton systems provides observational constraints to theories and models of the early dynamical evolution and migration history of planetary systems. Wright et al. (2009) showed that while the eccentricity distribution of planets in multiplanet systems is similar to that of apparently singleton systems, their semimajor axis distributions differ significantly. The concentration of planets with orbital periods near 3 days seen in the single-planet systems is absent in multiplanet systems, as is the sharp jump in planet frequency beyond $1 \mathrm{AU}$. The fact that these features (the three-day pileup and the $1 \mathrm{AU}$ jump) are functions planetary multiplicity strongly suggests that

\footnotetext{
7 Townes Fellow, Space Sciences Laboratory.

8 NSF Postdoctoral Fellow.

9 Prior to this, Wolszczan \& Frail (1992) detected the first planets outside the solar system: three extraordinary planets orbiting the pulsar PSR $1257+12$.
}

planet-planet interactions play a key role in migration and the origin of eccentricities.

\section{THE TENTH TRIPLE SYSTEM}

The announcement of HIP $14810 d$ herein marks the tenth system with three or more detected planets and only the sixth known to host three or more giant $\left(M \sin i>10 M_{\text {Earth }}\right)$ planets. ${ }^{10}$ The minimum masses and orbital periods of the planets in this system are similar to those of $v$ Andromedae, but with the inner and outermost components reversed. For the three planets of HIP 14810 we find: $M \sin i=3.9,1.2$, and 0.6 $M_{\mathrm{Jup}}$, and $P=6.67,147.7$, and 952 day, respectively. We find modest but significant eccentricities for all three components $(0.14,0.16$, and 0.17 , respectively).

Table 1 contains a summary of the stellar properties of HIP $14810(=\mathrm{BD}+20518)$, which sits at $53 \mathrm{pc}(\pi=18.7 \pm 1.3$; van Leeuwen 2009) and has $V=8.5$. We have performed an LTE analysis of our template spectra for HIP 14810 and derived its mass and radius using the methods described in Valenti \& Fischer (2005). Although HIP 14810 is a solar mass $\operatorname{star}\left(M=0.99 M_{\odot}\right)$, its metallicity $([\mathrm{Fe} / \mathrm{H}]=+0.26)$ and evolutionary status $\left(\Delta M_{\mathrm{V}}=0.63 \mathrm{mag}\right.$, as calculated in Wright (2004)) give it a spectral type of G5. Its low rotation $\left(v \sin i \sim 0.5 \pm 0.5 \mathrm{~km} \mathrm{~s}^{-1}\right.$ ) and Ca II $\mathrm{H} \& \mathrm{~K}$ activity levels ( $S=0.16$, measured with the methods described in Wright et al. (2004)) are consistent with it being an old star (age $\sim 8$ Gyr). This combined with its relatively small distance from the main sequence make it a particularly good radial velocity target, since it is expected to exhibit very low levels of jitter (Wright 2005). ${ }^{11}$

\footnotetext{
10 The others are $v$ Andomedae, 55 Cnc, $\mu$ Arae, HD 69830 (Lovis et al. 2006), and HD 37124 (Vogt et al. 2005).

11 We expect, based on hundreds of similar stars, $2 \mathrm{~m} \mathrm{~s}^{-1}$ of jitter, consistent with the residuals to our best fit. This value is somewhat lower than predicted by the formulae in Wright (2005) because that work included instrumental sources of noise associated with the HIRES CCD detector in place prior to 2004 August. The new detector has significantly better charge transfer properties, and apparently contributes a negligible amount to our overall error budget.
} 
Table 1

Stellar Properties of HIP 14810

\begin{tabular}{lc}
\hline \hline \multicolumn{1}{c}{ Parameter } & Value \\
\hline Spectral type & $\mathrm{G} 5 \mathrm{~V}$ \\
R.A. & $03^{\mathrm{h}} 11^{\mathrm{m}} 14.230$ \\
Decl. & $+21^{\circ} 05^{\prime} 50^{\prime} .49$ \\
$B-V$ & 0.78 \\
$V$ & 8.52 \\
Distance $(\mathrm{pc})$ & $52.9 \pm 4.1$ \\
$M_{\mathrm{V}}$ & 4.9 \\
$T_{\mathrm{eff}}(\mathrm{K})$ & $5485 \pm 44$ \\
$\log g\left[\mathrm{~cm} \mathrm{~s}{ }^{2}\right]$ & $4.220 \pm 0.06$ \\
{$[\mathrm{Fe} / \mathrm{H}]$} & $+0.26 \pm 0.03$ \\
$v \sin i$ & $0.54 \pm 0.5 \mathrm{~km} \mathrm{~s}$ \\
Mass $\left(M_{\odot}\right)$ & $0.99 \pm 0.04$ \\
Radius $\left(R_{\odot}\right)$ & $1.0 \pm 0.06$ \\
$S$ & 0.16 \\
$\log R_{\mathrm{HK}}^{\prime}$ & -5.01 \\
$\Delta M_{\mathrm{V}}(\mathrm{mag})$ & 0.64 \\
\hline
\end{tabular}

\section{VELOCITIES AND ORBITAL SOLUTION}

We began observations of HIP 14810 in 2005 as part of the N2K survey (Fischer et al. 2005) at Keck Observatory using HIRES (Vogt et al. 1994) and our usual iodine technique (Butler et al. 1996) to achieve typical internal (random) errors of 0.8$1.4 \mathrm{~m} \mathrm{~s}^{-1}$. The presence of the innermost planet and the large resuiduals to its orbital fit inspired the California Planet Search consortium to continue regular observations of this system at Keck.

Wright et al. (2007) announced ${ }^{12}$ the inner two planets of HIP 14810, though with a rather poor fit for the $c$ component due to the poor phase coverage and the unaccounted-for effects of the $d$ component. Also complicating the fit was what is now obviously a spurious data point, acquired during early dusk when significant contamination from the solar spectrum likely produced an erroneous radial velocity measurement. We have applied a more rigorous data retention scheme (based solely on the measured internal errors, ${ }^{13}$ not deviations from a fit) to the data set presented in Table 2. These velocities and uncertainties supersede our previously published values for this star, as we continue to refine our data reduction pipeline (Wright et al. 2009). Note that the times given in the table are in heliocentric Julian days, and the quoted errors are our internal (random) errors, with no "jitter" included.

By 2007, residuals to a two-planet fit clearly showed coherent structure indicative of an outer companion. As Figure 1 shows, by late 2008 , these residuals appeared to describe one complete orbit of a $P \sim 950 \mathrm{~d}$ planet with modest eccentricity $(e \sim 0.2)$. We have performed a Monte Carlo false alarm probability (FAP) analysis of the complete set of residuals to determine the likelihood that an orbital fit of this quality could have been arrived at by chance. This method is very similar to the FAP analyses in Butler et al. (2006), Wright et al. (2007), Butler et al. (2009), and Howard et al. (2009), and we refer the reader to those works for more details. After binning the data in $24 \mathrm{hr}$ intervals and subtracting the best two-planet fit, we redrew these residuals 1000 times (that is, we kept the times of observation the same for each trial, but at each time assigned a new velocity and

\footnotetext{
12 A preliminary orbit for the $b$ component also appears in the Catalog of Nearby Exoplanets (Butler et al. 2006).

13 We derive radial velocity measurements from each of many independent "chunks" of spectrum for each observation. Our internal error for a given observation is determined from the variance of these velocities (Wright 2005).
}

Table 2

Radial Velocities for HIP 14810

\begin{tabular}{|c|c|c|}
\hline $\begin{array}{c}\text { HJD } \\
\text { JD-2440000 }\end{array}$ & $\begin{array}{c}\text { Velocity } \\
\mathrm{m} \mathrm{s}^{-1}\end{array}$ & $\begin{array}{c}\text { Uncertainty } \\
\mathrm{m} \mathrm{s}^{-1}\end{array}$ \\
\hline 13693.76627 & -147.5 & 1.1 \\
\hline 13694.83716 & -489.3 & 1.2 \\
\hline 13695.91489 & -242.3 & 1.1 \\
\hline 13723.79085 & 146.4 & 1.0 \\
\hline 13724.69303 & 304.8 & 1.2 \\
\hline 13746.81748 & -13.1 & 1.1 \\
\hline 13747.85573 & -450.0 & 0.9 \\
\hline 13748.73690 & -448.5 & 1.2 \\
\hline 13749.74186 & -86.0 & 1.1 \\
\hline 13751.90068 & 342.3 & 1.0 \\
\hline 13752.80978 & 225.5 & 0.8 \\
\hline 13753.69384 & -96.2 & 1.1 \\
\hline 13753.81261 & -154.0 & 1.0 \\
\hline 13753.90329 & -195.5 & 1.2 \\
\hline 13775.83626 & -256.0 & 0.9 \\
\hline 13776.81286 & 107.0 & 1.2 \\
\hline 13777.72301 & 332.0 & 1.2 \\
\hline 13778.72061 & 398.1 & 1.1 \\
\hline 13779.74412 & 220.8 & 1.2 \\
\hline 13961.13028 & -293.8 & 1.1 \\
\hline 13962.13341 & -426.9 & 1.0 \\
\hline 13981.97179 & -492.7 & 1.1 \\
\hline 13982.94950 & -217.1 & 1.2 \\
\hline 13983.98363 & 137.6 & 1.0 \\
\hline 13984.09915 & 169.9 & 1.2 \\
\hline 13984.98803 & 333.0 & 1.1 \\
\hline 13985.10437 & 343.6 & 1.2 \\
\hline 14023.96949 & 88.2 & 1.3 \\
\hline 14047.98188 & -396.5 & 1.2 \\
\hline 14129.79605 & -208.9 & 0.9 \\
\hline 14130.74873 & 125.2 & 0.9 \\
\hline 14131.84182 & 334.8 & 1.2 \\
\hline 14138.80623 & 336.4 & 1.2 \\
\hline 14307.13007 & 92.8 & 1.0 \\
\hline 14336.07711 & -436.4 & 1.0 \\
\hline 14344.03162 & 34.5 & 1.0 \\
\hline 14345.14559 & 306.4 & 0.9 \\
\hline 14396.82488 & -108.0 & 1.0 \\
\hline 14397.91112 & 254.0 & 1.0 \\
\hline 14398.88586 & 411.3 & 1.1 \\
\hline 14399.89293 & 364.3 & 1.4 \\
\hline 14428.00013 & -184.5 & 1.0 \\
\hline 14428.87298 & -470.7 & 1.0 \\
\hline 14492.75897 & 356.1 & 0.9 \\
\hline 14544.74216 & 260.4 & 1.1 \\
\hline 14674.09015 & 219.2 & 1.2 \\
\hline 14718.05894 & 146.8 & 1.1 \\
\hline 14723.08578 & -434.2 & 1.0 \\
\hline 14725.92426 & 348.5 & 1.2 \\
\hline 14727.05509 & 271.3 & 1.0 \\
\hline 14727.97892 & -45.6 & 1.1 \\
\hline 14777.99836 & 67.5 & 1.1 \\
\hline 14805.83497 & 358.4 & 1.1 \\
\hline
\end{tabular}

velocity uncertainty pair randomly drawn from the entire set, with replacement). We then added the nominal orbital solution for the $c$ component back into these new residuals and performed a thorough search for the best-fit two-planet orbital solution to each of these 1000 realizations of the data. We measure the FAP as the fraction of these realizations for which we find a solution superior or equivalent in fit quality to the nominal solution.

Note that this is an extremely conservative test of the FAP of the new planet, $d$, because we have not restricted the parameter 

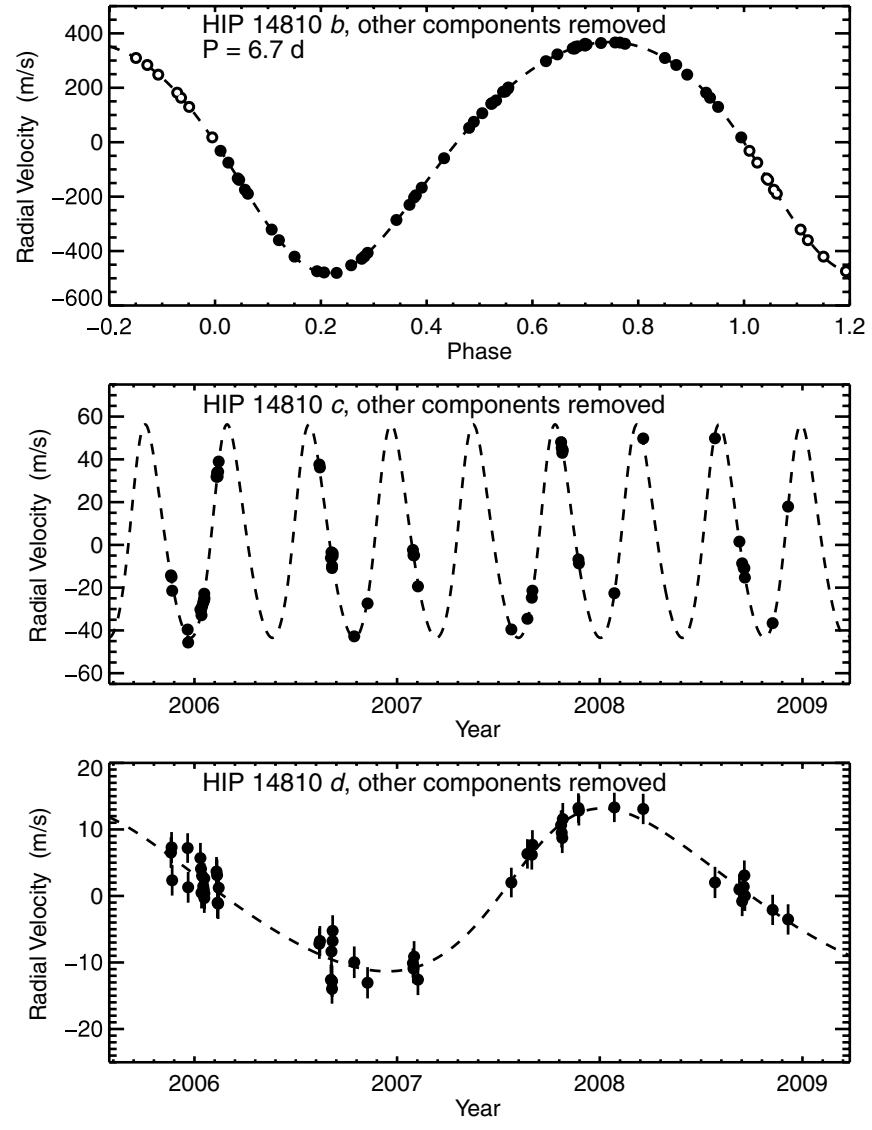

Figure 1. Radial velocity curves for the HIP 14810 triple system.

search for the FAP trials to long-period or low-eccentricity planets. The best-fit solutions found for the artificial data sets are thus often at short periods $(P<3$ day) and/or high eccentricites $e>0.7$ (see the discussion in Butler et al. 2009). Nonetheless, even with this large parameter space available, we find that none of our 1000 realizations produced a better fit than the actual data, yielding an FAP $<0.1 \%$.

We have fit the data using the publicly available multiplanet RV-fitting IDL package RV_FIT_MP, described in Wright \& Howard (2009). In Table 3 we present the best three-planet Keplerian (kinematic) fit, which yields rms residuals of $2.3 \mathrm{~m}$ $\mathrm{s}^{-1}$, and we plot the fit and velocities in Figure 1. We have observed just over one complete orbit for the outer component, HIP $14810 d$, and so its orbital parameters are sensitive to the assumption that there are no additional, external planets detectably influencing the velocities. In particular, it is possible that such an additional planet could be contributing a nearly linear trend to the observed radial velocities, a trend which might be absorbed into the $d$ component's orbital parameters as an inflated eccentricity. Any such degeneracy will be broken in the near future as the $d$ component completes its second orbit. Continued observation of this system will thus reveal the presence of any additional detectable planets and allow for further analysis of their interactions (e.g., Ford 2005; Wright et al. 2008).

\section{DYNAMICAL MODELING AND STABILITY ANALYSIS}

We have performed long-term numerical integrations to test for stability of the orbital solution here, under the assumption
Table 3

Orbital Elements for Exoplanets in the HIP 14810 System

\begin{tabular}{lccc}
\hline \hline \multicolumn{1}{c}{ Property } & $b$ & $c$ & $d$ \\
\hline $\mathrm{P}($ day $)$ & $6.673855(19)$ & $147.730(65)$ & $952(15)$ \\
$T_{0}(\mathrm{JD}-2440000)$ & $13694.5980(70)$ & $14672.2400(73)$ & $14317.1980(73)$ \\
$\mathrm{e}$ & $0.14270(94)$ & $0.164(12)$ & $0.173(37)$ \\
$\omega\left({ }^{\circ}\right)$ & $159.32(38)$ & $329.0(2.5)$ & $286(19)$ \\
$K\left(\mathrm{~m} \mathrm{~s}^{-1}\right)$ & $424.48(44)$ & $50.01(46)$ & $12.03(49)$ \\
$M \sin i\left(M_{\text {Jup }}\right)$ & $3.88(32)$ & $1.28(10)$ & $0.570(52)$ \\
$a(\mathrm{AU})$ & $0.0692(40)$ & $0.545(31)$ & $1.89(11)$ \\
& & & \\
\hline rms $\left(\mathrm{m} \mathrm{s}^{-1}\right)$ & & 2.3 & \\
$\chi_{v}^{2}$ & & 1.01 & \\
jitter $\left(\mathrm{m} \mathrm{s}^{-1}\right)$ & & 2 & \\
$N_{\text {obs }}$ & & 53 & \\
\hline
\end{tabular}

Notes. For succinctness, we express uncertainties using parenthetical notation, where the least significant digit of the uncertainty, in parentheses, and that of the quantity are to be understood to have the same place value. Thus, " $0.100(20)$ " indicates " $0.100 \pm 0.020$," " $1.0(2.0)$ " indicates " $1.0 \pm 2.0$," and " $1(20)$ " indicates " $1 \pm 20$."

that there are only three planets in the system. For these longterm stability tests, we applied direct $n$-body integrations to 100 orbital solutions consistent with the RV data, assuming an edge-on orientation. ${ }^{14}$ We integrated for at least $10^{8}$ years using the hybrid integrator in Mercury (Chambers 1999). For the majority of each integration, Mercury uses a mixed-variable symplectic integrator (Wisdom \& Holman 1991) with a time step approximately equal to a hundredth of the Keplerian orbital period calculated at a semimajor axis equal to the pericenter distance of the closest planet. During close encounters, Mercury uses a Bulrich-Stoer integrator with an accuracy parameter of $10^{-10}$. We identified each set of initial conditions as an unstable system if (1) two planets collide, (2) a planet is accreted onto the star (astrocentric distance less than $0.005 \mathrm{AU}$ ), or (3) a planet is ejected from the system (astrocentric distance exceeds $100 \mathrm{AU})$.

All of our simulations proved stable, and we manually verified that in all cases the final orbits were qualitatively similar to the initial conditions. We also ran an additional set of simulations for inclined (but coplanar) orientations of the system (i.e., smaller values of $\sin i$, and thus higher true planet masses and larger planet-planet interactions) and find the system to be stable for all tested scenarios with $i>3^{\circ}$.

In addition, we performed dynamical analyses of three planet solutions using ensembles of initial conditions generated via Bayesian posterior sampling methods (e.g., Ford 2006), both ignoring and including the planet-planet interactions. We found that the superposition of Keplerians approximation is a very good approximation for the nominal edge-on configuration. As the orbits approach face-on, the planet-planet interactions eventually become significant for inclinations of a few degrees. While we only considered coplanar configurations, we expect that our results are likely representative for relative inclinations of up to $20^{\circ}$ or $30^{\circ}$.

\footnotetext{
${ }^{14}$ We have estimated the uncertainties in the orbital parameters in Table 3 using a variant of the bootstrapping described in Butler et al. (2006). We generate a suite of plausible data sets and calculate the best-fit solution for each realization. We selected 100 of these realizations as the basis for this stability analysis, as in Wright et al. (2009).
} 


\section{PHOTOMETRIC OBSERVATIONS}

We acquired 1099 good photometric observations of HIP 14810 during four observing seasons spanning 1180 days between 2005 November and 2009 February with the T11 $0.8 \mathrm{~m}$ automated photometric telescope (APT) at Fairborn Observatory. The T11 APT and its two-channel Strömgren $b$ and $y$ photometer are very similar to the T8 $0.8 \mathrm{~m}$ APT and precision photometer described in Henry (1999).

The measurements of HIP 14810 were made differentially with respect to the comparison star HD $18404(V=5.80$, $B-V=0.42$, F5 IV). We combined the Strömgren $b$ and $y$ differential magnitudes into a single $(b+y) / 2$ passband to improve the precision of each measurement. Henry (1999) gives further details on the acquisition, reduction, and calibration of the APT data.

The standard deviation of a single observation from the mean of the entire data set is $0.00158 \mathrm{mag}$, which closely matches the typical measurement precision with this APT. Periodogram analysis of the full data set finds no significant periodicities between one and several hundred days. In particular, leastsquares sine fits to the 6.673855 and 147.73 day orbital periods of the inner $(b)$ and middle $(c)$ planets yield semiamplitudes of only $0.00008 \pm 0.00006$ and $0.00021 \pm 0.00006 \mathrm{mag}$, respectively, confirming that stellar activity is not the cause of the radial-velocity variations at these two periods. Although we do not expect radial velocity variations due to long period variations in stellar activity in such old stars (Wright et al. 2008), we have also searched for photometric variations at the period of the outer $(d)$ planet ( $P=952$ day). While our data set is not yet long enough to make this determination definitively, we see no suggestion of photometric variations at this period so far.

In the top panel of Figure 2, we plot the APT brightness measurements of HIP 14810 against phases computed from the 6.673855 day orbital period of the inner planet and a time of mid transit, JD 2,453,693.5856 \pm 0.0022 , predicted from the orbital elements in Table 3. The observations near the predicted time of transit are replotted with an expanded horizontal scale in the bottom panel of Figure 2. The solid curve shows the depth $(0.01 \mathrm{mag})$ and duration $( \pm 0.0125$ phase units) of a central transit of planet $b$, computed from the orbital elements and the planetary and stellar properties. The precisely determined orbit of HIP $14810 b$ (Figure 1, top panel) translates into an uncertainty in the mid-transit time that is smaller than the plotted points in the bottom of Figure 2. The mean of the 120 observations within the transit window is $2.81423 \pm 0.00015 \mathrm{mag}$; the mean of the 979 observations outside the window is $2.81410 \pm 0.00005 \mathrm{mag}$. Thus, the brightness levels inside and outside of the predicted transit window agree to within $0.00013 \mathrm{mag}$, allowing us to rule out transits of the $b$ component for all reasonable densities.

\section{CONCLUSIONS}

HIP 14810 is orbited by at least three giant planets, the outermost of which (HIP $14810 d$ ) has completed just over one orbit since we began monitoring it at Keck Observatory as part of the N2K project in 2005. This makes it only the sixth system known to host more than two giant $\left(M \sin i>10 M_{\text {Earth }}\right)$ planets. In retrospect, the previously published orbital solution for the middle $(c)$ component was hampered by a spurious data point, poor phase coverage, and the unaccounted-for effects of


Figure 2. Top panel: the 1099 photometric observations of HIP 14810 in the combined Strömgren $(b+y) / 2$ passband, acquired with the T11 $0.8 \mathrm{~m}$ APT over four observing seasons and plotted modulo the 6.673855-day orbital period of the inner planet HIP $14810 \mathrm{~b}$. Phase 0.0 corresponds to the predicted time of mid transit. A least-squares sine fit at the orbital period yields a semiamplitude of only $0.00008 \pm 0.00006$ mag. Bottom panel: the photometric observations of HD 14810 near the predicted time of transit replotted with an expanded scale on the abscissa. The solid curve shows the depth $(0.01 \mathrm{mag})$ and duration $( \pm 0.0125$ phase units) of a central transit, computed from the orbital elements and the planetary and stellar properties. The uncertainty in the predicted transit time is smaller that the diameter of the plotted points in the lower panel. The brightness levels of HIP 14810 within and outside the transit window agree to $0.00013 \mathrm{mag}$. Therefore, transits of HIP $14810 b$ are ruled out for all reasonable densities.

the $d$ component. Continued monitoring will reveal the existence of any additional outer planets.

We have performed a dynamical analysis for a suite of orbital solutions assuming no fourth planet, and find the system to be stable at nearly any inclination $\left(i<3^{\circ}\right)$. We have performed a photometric survey and find that the $b$ component does not transit.

The work herein is based on observations obtained at the W. M. Keck Observatory, which is operated jointly by the University of California and the California Institute of Technology. The Keck Observatory was made possible by the generous financial support of the W. M. Keck Foundation. We wish to recognize and acknowledge the very significant cultural role and reverence that the summit of Mauna Kea has always had within the indigenous Hawaiian community. We are most fortunate to have the opportunity to conduct observations from this mountain.

J.T.W. received support from NSF grant AST-0504874. G.W.M. received support from NASA grant NNG06AH52G, and D.A.F. from NASA grant NNG05G164G and the Cottrell Science Scholar Program. G.W.H. acknowledges support from NASA, NSF, Tennessee State University, and the State of Tennessee through its Centers of Excellence program. A.W.H. gratefully acknowledges support from a Townes Postdoctoral Fellowship at the UC Berkeley Space Sciences Laboratory. E.B.F. acknowledges the support of NASA RSA 1326409, and E.B.F. and D.V. acknowledge support of NASA grant NNX09AB35G. J.A.J. is supported by NSF grant AST-0702821.

We thank the anonymous referee for a helpful review. We acknowledge the University of Florida High-Performance Computing Center for providing computational resources and sup- 
port that have contributed to the results reported within this paper. This research has made use of NASA's Astrophysics Data System. This research has made use of the SIMBAD database, operated at CDS, Strasbourg, France

Facility Keck:I

\section{REFERENCES}

Butler, R. P., Howard, A. W., Vogt, S. S., \& Wright, J. T. 2009, ApJ, 691, 1738 Butler, R. P., Marcy, G. W., Fischer, D. A., Brown, T. M., Contos, A. R., Korzennik, S. G., Nisenson, P., \& Noyes, R. W. 1999, ApJ, 526, 916

Butler, R. P., Marcy, G. W., Williams, E., McCarthy, C., Dosanjh, P., \& Vogt, S. S. 1996, PASP, 108, 500

Butler, R. P., et al. 2006, ApJ, 646, 505

Chambers, J. E. 1999, MNRAS, 304, 793

Fischer, D. A., et al. 2005, ApJ, 620, 481

Fischer, D. A., et al. 2008, ApJ, 675, 790

Ford, E. B. 2005, AJ, 129, 1706

Ford, E. B. 2006, in ASP Conf. Ser. 352, New Horizons in Astronomy: Frank N. Bash Symposium, ed. S. J. Kannappan, S. Redfield, J. E. Kessler-Silacci, M. Landriau, \& N. Drory (San Francisco, CA: ASP), 15

Ford, E. B., Lystad, V., \& Rasio, F. A. 2005, Nature, 434, 873
Henry, G. W. 1999, PASP, 111, 845

Howard, A. W., et al. 2009, ApJ, 696, 75

Kley, W., Peitz, J., \& Bryden, G. 2004, A\&A, 414, 735

Lovis, C., et al. 2006, Nature, 441, 305

Mayor, M., et al. 2009, A\&A, submitted

Pepe, F., et al. 2007, A\&A, 462, 769

Rivera, E. J., et al. 2005, ApJ, 634, 625

Valenti, J. A., \& Fischer, D. A. 2005, ApJS, 159, 141

van Leeuwen, F. 2009, A\&A, 497, 209

Vogt, S. S., et al. 1994, Proc. SPIE, 2198, 362

Vogt, S. S., Butler, R. P., Marcy, G. W., Fischer, D. A., Henry, G. W., Laughlin, G., Wright, J. T., \& Johnson, J. A. 2005, ApJ, 632, 638

Wisdom, J., \& Holman, M. 1991, AJ, 102, 1528

Wolszczan, A., \& Frail, D. A. 1992, Nature, 355, 145

Wright, J. T. 2004, AJ, 128, 1273

Wright, J. T. 2005, PASP, 117, 657

Wright, J. T., \& Howard, A. W. 2009, ApJS, 182, 205

Wright, J. T., Marcy, G. W., Butler, R. P., \& Vogt, S. S. 2004, ApJS, 152, 261

Wright, J. T., et al. 2007, ApJ, 657, 533

Wright, J. T., Marcy, G. W., Butler, R. P., Vogt, S. S., Henry, G. W., Isaacson, H., \& Howard, A. W. 2008, ApJ, 683, L63

Wright, J. T., Upadhyay, S., Marcy, G. W., Fischer, D. A., Ford, E. B., \& Johnson, J. A. 2009, ApJ, 693, 1084 